\section{A CASE OF UTERINE INERTIA IN JAGUAR (PANTHERA ONCA)}

\author{
M. Navin Kumar ${ }^{1}$ and Md. Vikaruddin Ali Akbar² \\ ${ }^{1}$ Assistant Director, ${ }^{2}$ Veterinary Assistant Surgeon, \\ Nehru Zoological Park, Bahadurpura, Hyderabad, Andhra Pradesh, \\ India.
}

Dystokia is a common problem in cattle and in most of the other domestic animals due to various factors, one of them being malpresentation of foetus. However, there are hardly any reports of dystokia or of uterine inertia in wild felids on record. Therefore, a case of uterine inertia in captive jagaur at the Nehru Zoological Park is communicated.

The Nehru Zoological Park, Hyderabad acquired a pair of captive born Jaguars from Singapore Zoological Garden on 14 March 1993. The male was nine years old and the female was two years old. The female had given birth five times previously with no difficulty during which the gestation period varied from 99 to 105 days.

In the last gestation ( $6^{\text {th }}$ time $)$ on day 102 , the female delivered three weaklings. There was an intermittent straining with no progression in labour, hence it was suspected that she possessed one or more undelivered foetus inside. Accordingly the animal was darted with $8 \mathrm{mg}$ of valethamate bromide (Epidosin) to relax the cervix and with pituitrin (Oxytocin) 4 iu intramuscularly. The animal was left undisturbed for about half-an-hour and observed. The animal was still straining and the head of the foetus was visible. After another half-hour the animal was totally exhausted and no straining was observed. It was decided to correct the malpresentation of foetus manually.

The animal was tranquilized with blow pipe dart with a combination of $100 \mathrm{mg}$ xylazine and $50 \mathrm{mg}$ ketamine at about 1600 hours on 16 July 2000. When the animal became fully unconscious, it was confirmed that the foetus was dead and the uterus had reached the stage of inertia. We also tried to correct and retrieve the foetus, but due to inertia and total fluid loss, only the head was detached from the dead foetus and rest of it was left inside. Since, the animal was beginning to regain consciousness, and as it was not advisable to prolong anesthesia, the procedure was postponed. Before the animal completely gained consciousness about $200 \mathrm{ml}$ of rintose was administered intravenously, along with broad-spectrum antibiotics, analgesics and tetanus toxoid injections (intramuscularly). Early next morning the animal was noticed retaining the rest of the dead foetus inside without any effort of straining. Hence injections of pituitrin and valethamate bromide at the above mentioned doses were administered intramuscularly. One hour later the rest of the foetus was expelled. Although the animal looked active it did not consume anything until the next day. The diet of the animal was changed from beef to goat meat (about $1 \mathrm{~kg}$ per day, out of which it consumed only the $0.25 \mathrm{~kg}$ ). Injections of broad-spectrum antibiotics, analgesics and liver extracts were administered intramuscularly. As the animal went off-feed intermittently, the diet was altered to live baits of rabbits, chickens, and also chicken heads and neck which she consumed.

The treatment was continued up to 31 July 2000 and the animal was kept under observation. There was regular progress in her food consumption and finally the animal recovered completely.

\section{Acknowledgements}

Authors sincerely thank the management and animal keepers of Nehru Zoological Park, Hyderabad, for their everwilling cooperation in treating the case. 